study. Study participants were linked by a geographic information system (GIS) to characteristics in their neighborhood in order to spatially model the factors that contribute to increased psychological symptom severity at 3 months postinjury. Factor analysis identified underlying constructs of 32 neighborhood predictors, which were entered in a generalized estimating equation (GEE) regression analysis. Covariates included mechanism of injury, severity of injury, age, and health insurance.

Results Neighborhood characteristics loaded onto 4 constructs: neighborhood disconnectedness; concentrated disadvantage; crime, violence, and vacancy; and race/ethnicity. Higher PTSD symptom severity was reported by $36.8 \%$ and associated with neighborhood crime, violence, and vacancy, and higher depressive symptom severity was reported by $30.4 \%$ and associated with neighborhood disconnectedness. Both PTSD and depressive symptoms were associated with intentional mechanisms of injury, such as gunshot wounds, stabbing, and assault; and with having Medicaid or no insurance. Higher severity of injury was associated with depressive symptoms.

Conclusions Findings suggest that neighborhood characteristics such as neighborhood disconnectedness and crime, violence, and vacancy have measurable associations with psychological symptoms after injury. The evidence supports the need for interventions to improve post-injury mental health by modifying the urban environment.

\section{Equity and methods}

\section{IMPLICATIONS FOR HEALTH EQUITY AND LESSONS LEARNED FROM A COMMUNITY-ENGAGED MODIFIED DELPHI PROCESS AND NOMINAL GROUP TECHNIQUE}

\author{
1,2Kelsey Conrick, ${ }^{2}$ Brianna Mills, ${ }^{1,2}$ Frederick Rivara, ${ }^{1,2}$ Monica Vavilala. ${ }^{1}$ University of \\ Washington School of Social Work; ${ }^{2}$ University of Washington Harborview Injury Prevention \\ and Research Center
}

10.1136/injuryprev-2020-savir.40

Statement of Purpose Achieving injury-related health equity requires community-informed research and close collaboration among diverse (in lived experience, education, geography, etc.) stakeholders. Differing priorities, knowledge, and communication styles can inhibit participation in the consensus-building process, inhibiting the progress of research and development of successful interventions. Standardized, evidence-based procedures are needed to achieve consensus among diverse groups of stakeholders.

Methods/Approach We use a novel, rigorous process involving a combined Delphi process and Nominal Group Technique (NGT) to describe the development of recommendations for the pre-hospital care of the spine-injured athlete as a framework for achieving consensus among a group of stakeholders and community members. We outline our process and discuss lessons learned and implications for health equity.

Results We used an online modified Delphi process to achieve consensus on the research questions of priority to stakeholders. After conducting an independent systematic review, we presented our findings at an in-person meeting for twenty stakeholders. We used NGT to develop and agree upon protocol recommendations. Throughout this process, we learned several lessons. Most crucial to the success of the in-person NGT process is a reputable, experienced facilitator capable of managing complex group dynamics and adhering to agreed-upon rules of engagement. Clarification of research jargon and transparency throughout the research process engages stakeholders of all levels of experiences. Finally, innovative uses of technology may expedite the otherwise cumbersome process of providing opinions and voting to achieve consensus.

Conclusions While many researchers appreciate the value of community and stakeholder input towards the achievement of injury-related health equity, concern about managing and facilitating this process may limit its efficiency, inhibiting stakeholder investment in the research process.

Significance and Contributions to Injury and Violence Prevention Science Developing and implementing a well-informed, evidence-based protocol for achieving consensus may facilitate community-engaged research aimed to achieve injury-related health equity.

\section{Sports injury}

\section{CONCUSSION PREVENTION AWARENESS IN MIDDLE SCHOOL SPORTS}

Paula Gildner. University of North Carolina Injury Prevention Research Center

10.1136/injuryprev-2020-savir.41

Statement of Purpose Limited research specific to middle school (MS) sports on concussion prevention exists. We examined general perceptions, knowledge, attitudes, and communication constructs related to concussion prevention among MS sports stakeholders.

Methods/Approach Semi-structured interviews with coaches/ school staff $(n=6)$, parents, $(n=10)$ and athletes $(n=10)$ were conducted as part of a larger study throughout the 20182019 academic year at four North Carolina MSs. Interviews were transcribed verbatim; data were analyzed using the consensual qualitative tradition where a codebook was created using four progressive stages.

Results Three major themes were identified. First, there was a general lack of infrastructure and context surrounding sport safety and concussion prevention. Participants reported that the emphasis is on athletes simply exploring the varied sport options offered at the MS level, good sportsmanship and sport enjoyment; sport safety and concussion prevention was not a high concern. Second, there was limited awareness by stakeholders at the MS level concerning the importance of concussion prevention. Participants noted that concussion prevention is rarely discussed at the MS level and does not resonate with stakeholders. Third, there was ineffective communication between stakeholders regarding several topics, including concussion prevention. Participants noted that communication between the Athletic Directors, coaches, parents, and athletes was focused mainly on logistics, e.g., schedule changes. In addition, participants reported varied modes of communication utilized by school, of which most reportedly posed challenges for key stakeholders.

Conclusions These findings suggest a need to develop a cohesive strategy specific to the MS setting to disseminate concussion prevention information. Researchers and other MS sport stakeholders should collaborate to develop methods for sharing concussion prevention information at this level. 